Research Article

\title{
Complete Convergence for END Random Variables under Sublinear Expectations
}

\author{
Qunying Wu (i) \\ College of Science, Guilin University of Technology, Guilin 541004, China \\ Correspondence should be addressed to Qunying Wu; wqy666@glut.edu.cn
}

Received 1 February 2021; Revised 25 February 2021; Accepted 24 April 2021; Published 10 May 2021

Academic Editor: Maria Alessandra Ragusa

Copyright (c) 2021 Qunying Wu. This is an open access article distributed under the Creative Commons Attribution License, which permits unrestricted use, distribution, and reproduction in any medium, provided the original work is properly cited.

In this paper, the complete convergence theorems of partial sums and weighted sums for extended negatively dependent random variables in sublinear expectation spaces have been studied and established. Our results extend the corresponding results of classical probability spaces to the case of sublinear expectation spaces.

\section{Introduction}

The classical probability and statistics modeling is based on the linear basis of expectation and probability, and their important theoretical basis is the additivity of probability and expectation. However, in many application fields, because many uncertain factors can not be well solved, in order to better simulate this uncertainty phenomenon, we need new tools. The sublinear expectation space established by Peng ([1], [2]) belongs to this kind of tool. Sublinear expectation space is very useful in the simulation of uncertainty. They are widely used in statistics, finance, and insurance and have been studied by many probability statisticians since it came into being. For example, Peng ([1-5]) gave the basic structure, basic properties, and central limit theorem of sublinear expected space. Denis and Martini ([6]) studied a theoretical framework for the pricing of contingent claims in the presence of model uncertainty. Limit theorems for sublinear expectations have raised a large number of issues of interest recently. Marinacci ([7]) and Zhang (2016a [8], 2016b [9], 2016c [10]) established some inequalities and almost sure convergence theorems. Hu (2016 [11]), Chen (2016 [12]), Wu and Jiang (2018 [13]), and $\mathrm{Wu}$ and $\mathrm{Lu}(2020$ [14]) also studied strong limit theorem and law of the iterated logarithm.

The concept of complete convergence of sequence of random variables is introduced by Hsu and Robbins (1947 [15]). In view of Borel-Cantelli lemma, complete convergence means almost sure convergence. Therefore, complete convergence is one of the most important problems in probability limit theory. Many of its related results have been obtained in the probability space, for example, Wu (2010 [16]), Hu et al. (2012 [17]), Wang et al. (2014 [18]), Wang et al. (2015 [19]), Liu et al. (2015 [20]), Chen and Sung (2016 [21]), Tan et al. (2016 [22]), Wu and Jiang (2016 [23]), and Shen et al. (2017 [24]). Recently, Yu and Wu (2018 [25]), Wang and $\mathrm{Wu}$ (2019 [26]), and Deng and Wang (2020 [27]) obtained complete convergence theorem under sublinear expectations. In this paper, we study the complete convergence for extended negatively dependent random variables under sublinear expectation. As a result, the corresponding complete convergence theorems of the probability space have been generalized to the sublinear expectation space context.

Because sublinear expectation space relaxes the additivity of probability and expectation of traditional probability space and expands its application scope, and it is of great theoretical and practical significance to extend the limit theorem of traditional probability to space to sublinear expectation space. At the same time, because the sublinear expectation is nonadditive, the study of its limit theory becomes very complex and difficult.

\section{Related Concepts and Lemmas}

In the sublinear expectation space, many basic concepts need to be defined, such as sublinear expectation, capacity, independence, and identical distribution. This section gives a 
brief introduction to these concepts, which can be seen in detail in the works of Peng (1997 [1], 1999 [2]).

Let $(\Omega, \mathscr{F})$ be a given measurable space and let $\mathscr{H}$ be a linear space of real functions defined on $(\Omega, \mathscr{F})$ such that if $X_{1}, \ldots, X_{n} \in \mathscr{H}$, then $\varphi\left(X_{1}, \ldots, X_{n}\right) \in \mathscr{H}$ for each $\varphi \in C_{l, \text { Lip }}\left(\mathbb{R}_{n}\right)$, where $C_{l, \text { Lip }}\left(\mathbb{R}_{n}\right)$ denotes the linear space of local Lipschitz functions.

Definition 1. A sublinear expectation $\widehat{\mathbb{E}}$ on $\mathscr{H}$ is a function $\widehat{\mathbb{E}}: \mathscr{H} \longrightarrow[-\infty, \infty]$ satisfying the following properties: for all $X, Y \in \mathscr{H}$, we have

(i) Monotonicity: if $X \geq Y$, then $\widehat{\mathbb{E}} X \geq \widehat{\mathbb{E}} Y$

(ii) Constant preserving: $\widehat{\mathbb{E}} c=c$

(iii) Subadditivity: $\widehat{\mathbb{E}}(X+Y) \leq \widehat{\mathbb{E}} X+\widehat{\mathbb{E}} Y$

(iv) Positive homogeneity: $\widehat{\mathbb{E}}(\lambda X)=\lambda \widehat{\mathbb{E}} X, \lambda \geq 0$

The triple $(\Omega, \mathscr{H}, \widehat{\mathbb{E}})$ is called a sublinear expectation space.

The conjugate expectation $\widehat{\mathcal{E}}$ of $\widehat{\mathbb{E}}$ by $\widehat{\varepsilon} X:=-\widehat{\mathbb{E}}(-X), \forall X \in \mathscr{H}$.

Definition 2. Let $\mathscr{G} \subset \mathscr{F}$. A function $V: \mathscr{G} \longrightarrow[0,1]$ is called a capacity if

$$
\begin{aligned}
& V(\varnothing)=0, \\
& V(\Omega)=1, \\
& V(A) \leq V(B), \quad \text { for } \forall A \subseteq B, A, B \in \mathscr{G} .
\end{aligned}
$$

It is called to be subadditive if $V(A \cup B) \leq V(A)+V(B)$ for all $A, B \in \mathscr{G}$ with $A \cup B \in \mathscr{G}$. In the sublinear space $(\Omega, \mathscr{H}, \widehat{\mathbb{E}})$, we denote a pair $(\mathbb{V}, \nu)$ of capacities by

$$
\begin{aligned}
\mathbb{V}(A) & :=\inf \{\widehat{\mathbb{E}} \xi ; I(A) \leq \xi, \xi \in \mathscr{H}\}, \\
\nu(A) & :=1-\mathbb{V}\left(A^{c}\right), \quad \forall A \in \mathscr{F},
\end{aligned}
$$

where $I(\cdot)$ denotes an indicator function and $A^{c}$ is the complement set of $A$. By definition of $\mathbb{V}$ and $\nu$, it is obvious that $\mathbb{V}$ is subadditive, and $\nu(A) \leq \mathbb{V}(A)$ for any $A \in \mathscr{F}$.

The corresponding Choquet integrals/expectations $\left(C_{\mathbb{V}}, C_{\nu}\right)$ are defined by

$$
C_{V}(X):=\int_{0}^{\infty} V(X>x) \mathrm{d} x+\int_{-\infty}^{0}(V(X>x)-1) \mathrm{d} x,
$$

where $V$ is replaced by $\mathbb{V}$ and $\nu$, respectively.

Some basic useful properties of the sublinear expected space are given below, which are described in detail in the works of $\mathrm{Wu}$ and Jiang (2018 [13]).

\section{Proposition 1}

(i) For all $X, Y \in \mathscr{H}$,

$$
\begin{aligned}
\widehat{\varepsilon} X & \leq \widehat{\mathbb{E}} X, \\
\widehat{\mathbb{E}}(X+c) & =\widehat{\mathbb{E}} X+c, \\
|\widehat{\mathbb{E}}(X-Y)| & \leq \widehat{\mathbb{E}}|X-Y|, \\
\widehat{\mathbb{E}}(X-Y) & \geq \widehat{\mathbb{E}} X-\widehat{\mathbb{E}} Y .
\end{aligned}
$$

(ii) If $\widehat{\mathbb{E}} Y=\widehat{\varepsilon} Y$, then $\widehat{\mathbb{E}}(X+a Y)=\widehat{\mathbb{E}} X+a \widehat{\mathbb{E}} Y$ for $a$ ny $a \in \mathbb{R}$.

(iii)

$$
\begin{aligned}
& \widehat{\mathbb{E}} f \leq \mathbb{V}(A) \leq \widehat{\mathbb{E}} g, \\
& \widehat{\varepsilon} f \leq \nu(A) \leq \widehat{\varepsilon} g, \quad \text { if } f \leq I(A) \leq g, f, g \in \mathscr{H} .
\end{aligned}
$$

(iv) Markov inequality: $\forall X \in \mathscr{H}$ :

$$
\mathbb{V}(|X| \geq x) \leq \frac{\widehat{\mathbb{E}}\left(|X|^{p}\right)}{x^{p}}, \quad \forall x>0, p>0 .
$$

(v) Hö lder inequality: $\forall X, Y \in \mathscr{H}, p, q>1$, satisfying $p^{-1}+q^{-1}=1$ :

$$
\widehat{\mathbb{E}}(|X Y|) \leq\left(\widehat{\mathbb{E}}\left(|X|^{p}\right)\right)^{1 / p}\left(\widehat{\mathbb{E}}\left(|Y|^{q}\right)\right)^{1 / q} .
$$

(vi) Jensen inequality: $\forall X \in \mathscr{H}$ :

$$
\left(\widehat{\mathbb{E}}\left(|X|^{r}\right)\right)^{1 / r} \leq\left(\widehat{\mathbb{E}}\left(|X|^{s}\right)\right)^{1 / s} \quad \text { for } 0<r \leq s .
$$

Definition 3 (see $[2,8]$ )

(i) Identical distribution: let $X_{1}$ and $X_{2}$ be two random variables defined, respectively, in sublinear expectation spaces $\left(\Omega_{1}, \mathscr{H}_{1}, \widehat{\mathbb{E}}_{1}\right)$ and $\left(\Omega_{2}, \mathscr{H}_{2}, \widehat{\mathbb{E}}_{2}\right)$. They are called identically distributed if

$$
\widehat{\mathbb{E}}_{1}\left(\varphi\left(X_{1}\right)\right)=\widehat{\mathbb{E}}_{2}\left(\varphi\left(X_{2}\right)\right), \quad \forall \varphi \in C_{l, \text { Lip }}(\mathbb{R}),
$$

whenever the subexpectations are finite. A sequence $\left\{X_{n} ; n \geq 1\right\}$ of random variables is said to be identically distributed if, for each $i \geq 1, X_{i}$ and $X_{1}$ are identically distributed.

(ii) Extended negatively dependent: a sequence of random variables $\left\{X_{n} ; n \geq 1\right\}$ is said to be upper (resp. lower) extended negatively dependent if there is some dominating constant $K \geq 1$ such that

$$
\widehat{\mathbb{E}}\left(\prod_{i=1}^{n} \varphi_{i}\left(X_{i}\right)\right) \leq K \prod_{i=1}^{n} \widehat{\mathbb{E}}\left(\varphi_{i}\left(X_{i}\right)\right), \quad \forall n \geq 2,
$$

whenever the nonnegative functions $\varphi_{i}(x) \in C_{l, \text { Lip }}(\mathbb{R}), i=1,2, \ldots$, are all nondecreasing (resp. all nonincreasing). They are called extended negatively dependent if they are both upper extended negatively dependent and lower extended negatively dependent.

It is obvious that if $\left\{X_{n} ; n \geq 1\right\}$ is a sequence of upper (resp. lower) extended negatively dependent random variables and functions $f_{1}(x), f_{2}(x), \ldots \in C_{l, \text { Lip }}(\mathbb{R})$ are all nondecreasing (resp. all nonincreasing), then $\left\{f_{n}\left(X_{n}\right) ; n \geq 1\right\}$ is also a sequence of upper (resp. lower) extended negatively dependent random variables.

In the following, let $\left\{X_{n} ; n \geq 1\right\}$ be a sequence of random variables in $(\Omega, \mathscr{H}, \widehat{\mathbb{E}})$, and $S_{n}=\sum_{i=1}^{n} X_{i}$. The symbol $c$ stands for a generic positive constant which may differ from one 
place to another. Let $a_{n} \ll b_{n}$ denote that there exists a constant $c>0$ such that $a_{n} \leq c b_{n}$ for sufficiently large $n$.

To prove our results, we need the following two lemmas.

Lemma 1 (see Theorem 3.1 in [8]). Let $\left\{X_{k} ; k \geq 1\right\}$ be a sequence of upper extended negatively dependent random variables in $(\Omega, \mathscr{H}, \widehat{\mathbb{E}})$ with $\widehat{\mathbb{E}} X_{k} \leq 0$. Then, for any $x>0$,

$$
\mathbb{V}\left(S_{n} \geq x\right) \leq(1+K e) \frac{\sum_{k=1}^{n} \widehat{\mathbb{E}} X_{k}^{2}}{x^{2}}
$$

Lemma 2. Suppose $X \in \mathscr{H}, \alpha>0$.

(i) Then, for any $c>0$,

$$
C_{\mathbb{V}}\left(|X|^{2 / \alpha}\right)<\infty \Leftrightarrow \sum_{n=1}^{\infty} \mathbb{V}\left(|X|>c n^{\alpha / 2}\right)<\infty \Leftrightarrow \sum_{n=1}^{\infty} n \mathbb{V}\left(|X|>c n^{\alpha}\right)<\infty .
$$

(ii) If $C_{\mathbb{V}}\left(|X|^{2 / \alpha}\right)<\infty$, then, for any constant $c>0$ and integer $\theta>1$,

$$
\begin{gathered}
\sum_{k=1}^{\infty} \theta^{2 k} \mathbb{V}\left(|X|>c \cdot \theta^{\alpha k}\right)<\infty, \\
\lim _{n \longrightarrow \infty} n \mathbb{V}\left(|X|>c n^{\alpha / 2}\right)=0, \\
\lim _{n \longrightarrow \infty} n^{4 / 3} \mathbb{V}\left(|X|>c n^{2 \alpha / 3}\right)=0 .
\end{gathered}
$$

Proof

(i) For any $c>0$, it can be obtained by the definition of $C_{\mathbb{V}}\left(|X|^{2 / \alpha}\right)$ and the substitution of integral variables that

$$
\begin{aligned}
C_{\mathbb{V}}\left(|X|^{2 / \alpha}\right) & =\int_{0}^{\infty} \mathbb{V}\left(|X|>x^{\alpha / 2}\right) \mathrm{d} x \\
& =c^{2 / \alpha} \int_{0}^{\infty} \mathbb{V}\left(|X|>c y^{\alpha / 2}\right) \mathrm{d} y \quad\left(\text { let } y=c^{\alpha / 2} x\right) \\
& =2 c^{2 / \alpha} \int_{0}^{\infty} z \mathbb{V}\left(|X|>c z^{\alpha}\right) \mathrm{d} z . \quad\left(\text { let } z=y^{1 / 2}\right) .
\end{aligned}
$$

Because $\mathbb{V}\left(|X|>c y^{\alpha / 2}\right)$ is nonnegative and monotonically decreasing, according to the integral convergence method, we can obtain

$$
C_{\mathbb{V}}\left(|X|^{2 / \alpha}\right)<\infty \Leftrightarrow \sum_{n=1}^{\infty} \mathbb{V}\left(|X|>c n^{\alpha / 2}\right)<\infty .
$$

Also, note that

$\int_{0}^{\infty} z \mathbb{V}\left(|X|>c z^{\alpha}\right) \mathrm{d} z=\sum_{n=1}^{\infty} \int_{n-1}^{n} z \mathbb{V}\left(|X|>c z^{\alpha}\right) \mathrm{d} z$.

This implies that

$\frac{1}{2} \sum_{n=1}^{\infty} n \mathbb{V}\left(|X|>c n^{\alpha}\right) \leq \int_{0}^{\infty} z \mathbb{V}\left(|X|>c z^{\alpha}\right) \mathrm{d} z \leq 1+2 \sum_{n=1}^{\infty} n \mathbb{V}\left(|X|>c n^{\alpha}\right)$.
Hence,

$$
\int_{0}^{\infty} z \mathbb{V}\left(|X|>c z^{\alpha}\right) \mathrm{d} z<\infty \Leftrightarrow \sum_{n=1}^{\infty} n \mathbb{V}\left(|X|>c n^{\alpha}\right)<\infty .
$$

From combination (16), we get (12) setup.

(ii) Note that, for any integer $\theta>1$,

$$
\begin{aligned}
\sum_{n=1}^{\infty} n \mathbb{V}\left(|X|>c n^{\alpha}\right) & \geq \sum_{k=1}^{\infty} \sum_{\theta^{k-1} \leq n<\theta^{k}} \theta^{k-1} \mathbb{V}\left(|X|>c \theta^{\alpha k}\right) \\
& =\theta^{-2}(\theta-1) \sum_{k=1}^{\infty} \theta^{2 k} \mathbb{V}\left(|X|>c \theta^{\alpha k}\right) .
\end{aligned}
$$

Hence, by (12), $C_{\mathbb{V}}\left(|X|^{2 / \alpha}\right)<\infty$ implies (13). have

By $\int_{0}^{\infty} \mathbb{V}\left(|X|>c x^{\alpha / 2}\right) \mathrm{d} x<\infty$ and $\mathbb{V}\left(|X|>c x^{\alpha / 2}\right) \downarrow$, we

$$
\lim _{x \longrightarrow \infty} x \mathbb{V}\left(|X|>c x^{\alpha / 2}\right)=0 .
$$

This is equivalent to

$$
\lim _{x \longrightarrow \infty} x^{4 / 3} \mathbb{V}\left(|X|>c x^{2 \alpha / 3}\right)=0 .
$$

Therefore, (14) holds.

\section{Complete Convergence Theorems}

In the sublinear expectation space, we define that $X_{n} \longrightarrow X$ completely converges as follows.

Definition 4. A sequence of random variables $\left\{X_{n} ; n \geq 1\right\}$ is said to completely converge to $X$, denoted by $X_{n} \longrightarrow^{c} X$ as $n \longrightarrow \infty$ if $\sum_{n=1}^{\infty} \mathbb{V}\left(\left|X_{n}-X\right|>\varepsilon\right)<\infty$ for any $\epsilon>0$.

The purpose of this paper is to extend corresponding results from the probability space to sublinear expectation space. Our results are as follows. 
Theorem 1. Let $\left\{X_{n} ; n \geq 1\right\}$ be a sequence of upper extended negatively dependent and identically distributed random variables. Let, for some $0<\alpha \leq 1$,

$$
\begin{gathered}
\widehat{\mathbb{E}}\left(\left|X_{1}\right|^{2 / \alpha}\right) \leq C_{\mathbb{V}}\left(\left|X_{1}\right|^{2 / \alpha}\right)<\infty, \\
0<a_{n k} \leq c n^{-\alpha} \quad \text { for } k \leq n \\
\text { and some } 0<c<\infty, a_{n k}=0 \text { for } k>n, \\
c_{n}:=\sum_{k=1}^{n} a_{n k}^{2}=o\left(\log ^{-1} n\right) .
\end{gathered}
$$

Then,

$$
\sum_{n=1}^{\infty} \mathbb{V}\left(\sum_{k=1}^{n} a_{n k}\left(X_{k}-\widehat{\mathbb{E}} X_{k}\right)>\epsilon\right)<\infty \quad \text { for any } \epsilon>0 .
$$

Furthermore, if $\left\{X_{n} ; n \geq 1\right\}$ is extended negatively dependent, then

$$
\sum_{n=1}^{\infty} \mathbb{V}\left(\sum_{k=1}^{n} a_{n k}\left(X_{k}-\widehat{\varepsilon} X_{k}\right)<-\epsilon\right)<\infty \quad \text { for any } \epsilon>0 .
$$

In particular, if $\left\{X_{n} ; n \geq 1\right\}$ is extended negatively dependent and $\widehat{\mathbb{E}} X_{k}=\widehat{\varepsilon} X_{k}$, then

$$
\sum_{k=1}^{n} a_{n k}\left(X_{k}-\widehat{\mathbb{E}} X_{k}\right) \stackrel{c}{\longrightarrow} 0 \text {. }
$$

Remark 1. It is known by Lemma 4.5 (iii) in [9] that if $\lim _{c \rightarrow \infty} \widehat{\mathbb{E}}\left(|X|^{2 / \alpha}-c\right)^{+}=0$ or $\widehat{\mathbb{E}}$ is countably subadditive, then $\overrightarrow{\mathbb{E}}\left(\left|X_{1}\right|^{2 / \alpha}\right) \leq C_{\mathbb{V}}\left(\left|X_{1}\right|^{2 / \alpha}\right)$.

Theorem 2. Let $\left\{X_{n} ; n \geq 1\right\}$ be a sequence of extended negatively dependent and identically distributed random variables. Let, for some $\alpha>1$,

$$
C_{\mathbb{V}}\left(\left|X_{1}\right|^{2 / \alpha}\right)<\infty
$$

Then,

$$
\frac{S_{n}}{n^{\alpha}} \stackrel{c}{\longrightarrow} 0
$$

Remark 2. Theorems 1 and 2 extend the corresponding complete convergence results from the probability space to sublinear expectation space.

Proof of Theorem 1. By the Jensen inequality, $\widehat{\mathbb{E}} X_{1}^{2} \leq\left(\widehat{\mathbb{E}}\left|X_{1}\right|^{2 / \alpha}\right)^{\alpha}<\infty$ for $0<\alpha \leq 1$. Without loss of generality, we can assume that $\widehat{\mathbb{E}} X_{1}^{2}=1$.

For upper extended negatively dependent random variables $\left\{X_{n} ; n \geq 1\right\}$, in order to ensure that the truncated random variables are also upper extended negatively dependent, we need that truncated functions belong to $C_{l, \text { Lip }}$ and are nondecreasing. Let $f_{c}(x):=x I(x \leq c)+c I(x>c)$ for any $c>0$; for any $1 \leq k \leq n, n \geq 1$, let

$$
\begin{aligned}
X_{n k}^{(1)}:= & f_{n^{-\alpha / 3} a_{n k}^{-1}}\left(X_{k}\right)=X_{k} I\left(X_{k} \leq n^{-\alpha / 3} a_{n k}^{-1}\right) \\
& +n^{-\alpha / 3} a_{n k}^{-1} I\left(X_{k}>n^{-\alpha / 3} a_{n k}^{-1}\right), \\
X_{n k}^{(2)}:= & \left(X_{k}-n^{-\alpha / 3} a_{n k}^{-1}\right) I\left(X_{k}>\frac{\epsilon a_{n k}^{-1}}{4}\right), \\
X_{n k}^{(3)}:= & X_{k}-X_{n k}^{(1)}-X_{n k}^{(2)} \\
= & \left(X_{k}-n^{-\alpha / 3} a_{n k}^{-1}\right) I\left(n^{-\alpha / 3} a_{n k}^{-1}<X_{k}<\frac{\epsilon a_{n k}^{-1}}{4}\right) .
\end{aligned}
$$

Then, $\left\{X_{n k}^{(1)} ; 1 \leq k \leq n, n \geq 1\right\}$ is also a sequence of upper extended negatively dependent random variables by $f_{c}(x) \in C_{l, \text { Lip }}$ and $f_{c}(x)$ being nondecreasing. Define

$$
\begin{aligned}
& T_{n}^{(i)}:=\sum_{k=1}^{n} a_{n k} X_{n k}^{(i)}, \quad i=1,2,3, \\
& \widetilde{T}_{n}^{(1)}:=\sum_{k=1}^{n} a_{n k}\left(X_{n k}^{(1)}-\widehat{\mathbb{E}}\left(X_{n k}^{(1)}\right)\right) .
\end{aligned}
$$

Then,

$$
\sum_{k=1}^{n} a_{n k}\left(X_{k}-\widehat{\mathbb{E}} X_{k}\right)=\widetilde{T}_{n}^{(1)}+T_{n}^{(2)}+T_{n}^{(3)}+J_{n},
$$

where $J_{n}=\sum_{k=1}^{n} a_{n k}\left(\widehat{\mathbb{E}}\left(X_{n k}^{(1)}\right)-\widehat{\mathbb{E}} X_{k}\right)$.

Therefore, in order to prove (26), it suffices to verify that, for any $\epsilon>0$,

$$
\begin{gathered}
\sum_{n=1}^{\infty} \mathbb{V}\left(\widetilde{T}_{n}^{(1)}>\epsilon\right)<\infty, \\
\sum_{n=1}^{\infty} \mathbb{V}\left(T_{n}^{(i)}>\varepsilon\right)<\infty, \quad i=2,3, \\
J_{n} \longrightarrow 0 \text { as } n \longrightarrow \infty .
\end{gathered}
$$

It shall be noted that, in the probability space, there is an equality: $E I(|X| \leq a)=P(|X| \leq a)$; however, in the sublinear expectation space, $\widehat{\mathbb{E}}$ is defined through continuous functions in $C_{l, \text { Lip }}$ and the indicator function $I(|x| \leq a)$ may be discontinuous. Therefore, the expression $\widehat{\mathbb{E}} I(|X| \leq a)$ may not exist. This needs to modify the indicator function by functions in $C_{l, \mathrm{Lip}}$. To this end, we define the function $g(x) \in C_{l, \text { Lip }}(\mathbb{R})$ as follows.

For $0<\mu<1$, let $g(x) \in C_{l \text {,Lip }}(\mathbb{R})$ be a nonincreasing function such that $0 \leq g(x) \leq 1$ for all $x$ and $g(x)=1$ if $x \leq \mu$ and $g(x)=0$ if $x>1$. Then,

$$
\begin{aligned}
& I(x \leq \mu) \leq g(x) \leq I(x \leq 1), \\
& I(x>1) \leq 1-g(x) \leq I(x>\mu) .
\end{aligned}
$$

Note that 


$$
\begin{aligned}
\left|X_{k}-X_{n k}^{(1)}\right| & =\left(X_{k}-n^{-\alpha / 3} a_{n k}^{-1}\right) I\left(X_{k}>n^{-\alpha / 3} a_{n k}^{-1}\right) \\
& \leq\left|X_{k}\right| I\left(\left|X_{k}\right|>n^{-\alpha / 3} a_{n k}^{-1} \geq c n^{2 \alpha / 3}\right) \\
& \ll \frac{\left|X_{k}\right|^{2 / \alpha}}{n^{(2 \alpha(2 /(\alpha-1))) / 3}} .
\end{aligned}
$$

By (23), (24) and (36) follow that

$$
\begin{aligned}
\left|J_{n}\right| & \leq \sum_{k=1}^{n} a_{n k} \widehat{\mathbb{E}}\left|X_{k}-X_{n k}^{(1)}\right| \leq \sum_{k=1}^{n} a_{n k} \frac{\widehat{\mathbb{E}}\left|X_{1}\right|^{\alpha / 2}}{n^{(4 / 3)-(2 \alpha / 3)}} \\
& \ll n^{-(\alpha / 3)-(1 / 3)} \longrightarrow 0, \quad n \longrightarrow \infty .
\end{aligned}
$$

Now, we prove that (34). Let $\widetilde{X}_{n k}^{(1)}=X_{n k}^{(1)}-\widehat{\mathbb{E}} X_{n k}^{(1)}$. Then, $\widetilde{T}_{n}^{(1)}=\sum_{k=1}^{n} a_{n k} \tilde{X}_{n k}^{(1)}$. Fix $n \geq 1 . \quad$ Let $u=\min \left(\left(\epsilon / 8 c_{n}\right),\left(n^{\alpha / 3} / 2\right)\right)$. By $X_{n k}^{(1)} \leq n^{-\alpha / 3} a_{n k}^{-1},\left|X_{n k}^{(1)}\right| \leq\left|X_{k}\right|$, (24), and Jensen inequality, we obtain

$$
\begin{aligned}
u a_{n k} \tilde{X}_{n k}^{(1)} & \leq u a_{n k} X_{n k}^{(1)}+u a_{n k} \widehat{\mathbb{E}}\left|X_{n k}^{(1)}\right| \\
& \leq \frac{1}{2}+u a_{n k}\left(\widehat{\mathbb{E}} X_{1}^{2}\right)^{1 / 2} \leq \frac{1}{2}+\frac{c n^{-2 \alpha / 3}}{2} \leq 1 .
\end{aligned}
$$

It follows that

$$
\begin{aligned}
& \exp \left(u a_{n k} \widetilde{X}_{n k}^{(1)}\right)=\exp \left(u a_{n k} \widetilde{X}_{n k}^{(1)} I\left(u a_{n k} \tilde{X}_{n k}^{(1)}<-1\right)+u a_{n k} \widetilde{X}_{n k}^{(1)} I\left(\left|u a_{n k} \widetilde{X}_{n k}^{(1)}\right| \leq 1\right)\right) \\
& =\exp \left(u a_{n k} \tilde{X}_{n k}^{(1)} I\left(u a_{n k} \tilde{X}_{n k}^{(1)}<-1\right)\right) \exp \left(u a_{n k} \tilde{X}_{n k}^{(1)} I\left(\left|u a_{n k} \tilde{X}_{n k}^{(1)}\right| \leq 1\right)\right) \\
& \leq \begin{cases}1, & u a_{n k} \tilde{X}_{n k}^{(1)}<-1, \\
\exp \left(u a_{n k} \tilde{X}_{n k}^{(1)}\right), & \left|u a_{n k} \tilde{X}_{n k}^{(1)}\right| \leq 1,\end{cases} \\
& = \begin{cases}1+u a_{n k} \widetilde{X}_{n k}^{(1)}+\left|u a_{n k} \widetilde{X}_{n k}^{(1)}\right|, & u a_{n k} \widetilde{X}_{n k}^{(1)}<-1, \\
1+\sum_{j=1}^{\infty} \frac{\left(u a_{n k} \widetilde{X}_{n k}^{(1)}\right)^{j}}{j !}, & \left|u a_{n k} \widetilde{X}_{n k}^{(1)}\right| \leq 1,\end{cases} \\
& \leq \begin{cases}1+u a_{n k} \widetilde{X}_{n k}^{(1)}+u^{2} a_{n k}^{2}\left(\widetilde{X}_{n k}^{(1)}\right)^{2}, & u a_{n k} \widetilde{X}_{n k}^{(1)}<-1, \\
1+u a_{n k} \tilde{X}_{n k}^{(1)}+\frac{\left(u a_{n k} \widetilde{X}_{n k}^{(1)}\right)^{2}}{2}\left(1+2 \sum_{j=3}^{\infty} \frac{1}{j !}\right), & \left|u a_{n k} \widetilde{X}_{n k}^{(1)}\right| \leq 1,\end{cases} \\
& \leq 1+u a_{n k} \tilde{X}_{n k}^{(1)}+u^{2} a_{n k}^{2}\left(\tilde{X}_{n k}^{(1)}\right)^{2} \text {. }
\end{aligned}
$$

Hence, by $\widehat{\mathbb{E}} \tilde{X}_{n k}^{(1)}=0$, and $\widehat{\mathbb{E}}\left(\widetilde{X}_{n k}^{(1)}\right)^{2} \leq 2\left(\widehat{\mathbb{E}}\left(X_{n k}^{(1)}\right)^{2}+\right.$ $\left.\left(\widehat{\mathbb{E}} X_{n k}^{(1)}\right)^{2}\right) \leq 4 \widehat{\mathbb{E}}\left(X_{n k}^{(1)}\right)^{2} \leq 4 \widehat{\mathbb{E}} X_{1}^{2}=4$, we obtain

$$
\widehat{\mathbb{E}}\left(\exp \left(u a_{n k} \tilde{X}_{n k}^{(1)}\right)\right) \leq 1+4 u^{2} a_{n k}^{2} \leq \exp \left(4 u^{2} a_{n k}^{2}\right)
$$

Since $\left\{u a_{n k} \widetilde{X}_{n k}^{(1)}, n \geq 1, k \leq n\right\}$ is also upper extended negatively dependent, it follows from (25) that

$$
\begin{aligned}
\widehat{\mathbb{E}}\left(\exp \left(u \widetilde{T}_{n}^{(1)}\right)\right) & =\widehat{\mathbb{E}}\left(\prod_{k=1}^{n} \exp \left(u a_{n k} \widetilde{X}_{n k}^{(1)}\right)\right) \\
& \leq K \prod_{k=1}^{n} \widehat{\mathbb{E}}\left(\exp \left(u a_{n k} \widetilde{X}_{n k}^{(1)}\right)\right) \\
& \ll \prod_{k=1}^{n} \exp \left(4 u^{2} a_{n k}^{2}\right)=\exp \left(4 u^{2} c_{n}\right) .
\end{aligned}
$$

By the Markov inequality,

$$
\mathbb{V}\left(\widetilde{T}_{n}^{(1)}>\varepsilon\right) \leq \exp (-\epsilon u) \widehat{\mathbb{E}} \exp \left(u \widetilde{T}_{n}^{(1)}\right) \ll \exp \left(-\epsilon u+4 u^{2} c_{n}\right) .
$$

Let $\mathbb{N}_{1}:=\left\{n ;\left(\epsilon / 4 c_{n}\right)>n^{\alpha / 3}\right\}$ and $\mathbb{N}_{2}:=\left\{n ;\left(\epsilon / 4 c_{n}\right) \leq n^{\alpha / 3}\right\}$.

If $n \in \mathbb{N}_{1}$, i.e., $\left(\epsilon / 4 c_{n}\right)>n^{\alpha / 3}$, then, by the definition of $u$, we have $u=\left(n^{\alpha / 3} / 2\right)$ and $-\epsilon u+4 u^{2} c_{n} \leq\left(-\epsilon n^{\alpha / 3} / 4\right)$. Hence,

$$
\sum_{n \in \mathbb{N}_{1}} \mathbb{V}\left(\widetilde{T}_{n}^{(1)}>\epsilon\right) \ll \sum_{n=1}^{\infty} \exp \left(\frac{-\epsilon n^{\alpha / 3}}{4}\right)<\infty .
$$

If $n \in \mathbb{N}_{2}$, i.e., $\left(\epsilon / 4 c_{n}\right) \leq n^{\alpha / 3}$, then, by the definition of $u$, we have $u=\epsilon / 8 c_{n}$ and $-\epsilon u+4 u^{2} c_{n}=-\left(\epsilon^{2} / 16 c_{n}\right)$. Note that $c_{n}<\left(\epsilon^{2} / 32 \log n\right)$ for sufficiently large $n$ from $c_{n}=o\left(\log ^{-1} n\right)$. Hence,

$$
\sum_{n \in \mathbb{N}_{2}} \mathbb{V}\left(\widetilde{T}_{n}^{(1)}>\epsilon\right) \leq \sum_{n=1}^{\infty} \exp \left(-\frac{\epsilon^{2}}{16 c_{n}}\right) \ll \sum_{n=1}^{\infty} \frac{1}{n^{2}}<\infty .
$$

That is that (34) holds from

$$
\sum_{n=1}^{\infty} \mathbb{V}\left(\widetilde{T}_{n}^{(1)}>\epsilon\right)=\sum_{n \in \mathbb{N}_{1}} \mathbb{V}\left(\widetilde{T}_{n}^{(1)}>\right)+\sum_{n \in \mathbb{N}_{2}} \mathbb{V}\left(\widetilde{T}_{n}^{(1)}>\epsilon\right)<\infty .
$$

Next, we prove that $\sum_{n=1}^{\infty} \mathbb{V}\left(T_{n}^{(2)}>\epsilon\right)<\infty$. By $(24)$, 


$$
\left(T_{n}^{(2)}>\epsilon\right) \subset \bigcup_{k=1}^{n}\left(X_{k}>\frac{\epsilon a_{n k}^{-1}}{4}\right) \subset \bigcup_{k=1}^{n}\left(X_{k}>\epsilon n^{\alpha}(4 c)^{-1}:=b n^{\alpha}\right),
$$

where $b=\epsilon(4 c)^{-1}>0$. It shall be noted that the identical distribution is defined under $\widehat{\mathbb{E}}$, not under $\mathbb{V}$ (see Definition 3). $X_{i}$ identical distribution implies $\widehat{\mathbb{E}}\left(f\left(X_{i}\right)\right)=\widehat{\mathbb{E}}\left(f\left(X_{1}\right)\right)$ for $f(\cdot) \in C_{l, \text { Lip }}(\mathbb{R})$, but does not imply $\mathbb{V}\left(f\left(X_{i}\right) \in A\right)=\mathbb{V}\left(f\left(X_{1}\right) \in A\right)$. Therefore, in the calculation of $\mathbb{V}\left(f\left(X_{i}\right) \in A\right)$, we need to convert $\mathbb{V}$ to $\widehat{\mathbb{E}}$. Thus, by (5) and (37),

$$
\begin{aligned}
\mathbb{V}\left(T_{n}^{(2)}>\epsilon\right) & \leq \sum_{k=1}^{n} \mathbb{V}\left(X_{k}>b n^{\alpha}\right) \leq \sum_{k=1}^{n} \widehat{\mathbb{E}}\left(1-g\left(\frac{X_{k}}{b n^{\alpha}}\right)\right) \\
& =\sum_{k=1}^{n} \widehat{\mathbb{E}}\left(1-g\left(\frac{X_{1}}{b n^{\alpha}}\right)\right) \leq \sum_{k=1}^{n} \mathbb{V}\left(X_{1}>\mu b n^{\alpha}\right) \\
& \leq n \mathbb{V}\left(\left|X_{1}\right|>\mu b n^{\alpha}\right) .
\end{aligned}
$$

Therefore, from (12) and (23),

$$
\sum_{n=1}^{\infty} \mathbb{V}\left(T_{n}^{(2)}>\varepsilon\right) \leq \sum_{n=1}^{\infty} n \mathbb{V}\left(\left|X_{1}\right|>\mu b n^{\alpha}\right)<\infty .
$$

Lastly, we prove that $\sum_{n=1}^{\infty} \mathbb{V}\left(T_{n}^{(3)}>\epsilon\right)<\infty$. Since

$$
\begin{array}{r}
\left(T_{n}^{(3)}:=\sum_{k=1}^{n} a_{n k}\left(X_{k}-n^{-\alpha / 3} a_{n k}^{-1}\right) I\left(n^{-\alpha / 3} a_{n k}^{-1}<X_{k}<\frac{\epsilon a_{n k}^{-1}}{4}\right)>\varepsilon\right) \\
C\left(\sum_{k=1}^{n} a_{n k} X_{k} I\left(n^{-\alpha / 3} a_{n k}^{-1}<X_{k}<\frac{\epsilon a_{n k}^{-1}}{4}\right)>\epsilon\right) \\
\left.C \text { (there exist at least } 4 \text { indices } k \text { such that } X_{k}>a_{n k}^{-1} n^{-\alpha / 3}\right) .
\end{array}
$$

The last inclusion relation above holds that if there are only less than 4 indices $k$, such that $X_{k}>a_{n k}^{-1} n^{-\alpha / 3}$, then, in the sum $\sum_{k=1}^{n} a_{n k} X_{k} I\left(n^{-\alpha / 3} a_{n k}^{-1}<X_{k}<\left(\epsilon a_{n k}^{-1} / 4\right)\right)$, there are only less than 4 indices $k$, such that $a_{n k} X_{k} I\left(n^{-\alpha / 3} a_{n k}^{-1}<X_{k}<\left(\epsilon a_{n k}^{-1} / 4\right)\right) \neq 0$, and each term $a_{n k} X_{k} I\left(n^{-\alpha / 3} a_{n k}^{-1}<X_{k}<\left(\epsilon a_{n k}^{-1} / 4\right)\right)<(\epsilon / 4), \quad$ so $\sum_{k=1}^{n} a_{n k} X_{k} I\left(n^{-\alpha / 3} a_{n k}^{-1}<X_{k}<\left(\epsilon a_{n k}^{-1} / 4\right)\right)<\epsilon$. This is in contradiction with $\sum_{k=1}^{n} a_{n k} X_{k} I\left(n^{-\alpha / 3} a_{n k}^{-1}<X_{k}<\left(\epsilon a_{n k}^{-1} / 4\right)\right)>\epsilon$.

Therefore, by the definition of extended negatively dependent (5), (24), and (37), we have

$$
\begin{aligned}
\mathbb{V}\left(T_{n}^{(3)}>\varepsilon\right) & \leq \mathbb{V}\left(\text { there exist at least } 4 \text { indices } k \text { such that } a_{n k} X_{k}>n^{-\alpha / 3}\right) \\
& \leq \sum_{1 \leq i_{1}<\cdots<i_{4} \leq n} \mathbb{V}\left(a_{n i_{1}} X_{i_{1}}>n^{-\alpha / 3}, a_{n i_{2}} X_{i_{2}}>n^{-\alpha / 3}, a_{n i_{3}} X_{i_{3}}>n^{-\alpha / 3}, a_{n i_{4}} X_{i_{4}}>n^{-\alpha / 3}\right) \\
& \leq \sum_{1 \leq i_{1}<\ldots<i_{4} \leq n} \widehat{\mathbb{E}}\left(\prod_{j=1}^{4}\left(1-g\left(a_{n i_{j}} n^{\alpha / 3} X_{i_{j}}\right)\right)\right) \\
& \leq \sum_{1 \leq i_{1}<\ldots<i_{4} \leq n} K \prod_{j=1}^{4} \widehat{\mathbb{E}}\left(1-g\left(a_{n i_{j}} n^{\alpha / 3} X_{i_{j}}\right)\right) \\
& \leq \sum_{1 \leq i_{1}<\ldots<i_{4} \leq n} K \prod_{j=1}^{4} \hat{\mathbb{E}}\left(1-g\left(a_{n i_{j}} n^{\alpha / 3}\left|X_{i_{j}}\right|\right)\right) \\
& \ll \sum_{1 \leq i_{1}<\ldots<i_{4} \leq n}\left[\widehat{\mathbb{E}}\left(1-g\left(c n^{-2 \alpha / 3}\left|X_{1}\right|\right)\right)\right]^{4} \\
& \leq C_{n}^{4} \mathbb{V}^{4}\left(\left|X_{1}\right|>\mu c^{-1} n^{2 \alpha / 3}\right) \leq n^{4} \mathbb{V}^{4}\left(\left|X_{1}\right|>\mu c^{-1} n^{2 \alpha / 3}\right) .
\end{aligned}
$$

Thus,

$$
\sum_{n=1}^{\infty} \mathbb{V}\left(T_{n}^{(3)}>\epsilon\right) \ll \sum_{n=1}^{\infty} n^{-4 / 3}\left(n^{4 / 3} \mathbb{V}\left(\left|X_{1}\right|>\mu c^{-1} n^{2 \alpha / 3}\right)\right)^{4} \ll \sum_{n=1}^{\infty} n^{-4 / 3}<\infty
$$


from (14). Together with (50), we obtain (35). This completes the proof of (26).

Furthermore, if $\left\{X_{n} ; n \geq 1\right\}$ is extended negatively dependent, then $\left\{-X_{n} ; n \geq 1\right\}$; also, satisfying the conditions of Theorem 1 and considering $\left\{-X_{n} ; n \geq 1\right\}$ instead of $\left\{X_{n} ; n \geq 1\right\}$ in (26), for any $\epsilon>0$, we can obtain by $\widehat{\mathbb{E}}\left(-X_{k}\right)=-\widehat{\varepsilon} X_{k}$,

$$
\sum_{n=1}^{\infty} \mathbb{V}\left(\sum_{k=1}^{n} a_{n k}\left(-X_{k}-\widehat{\mathbb{E}}\left(-X_{k}\right)\right)>\epsilon\right)=\sum_{n=1}^{\infty} \mathbb{V}\left(-\sum_{k=1}^{n} a_{n k}\left(X_{k}-\widehat{\varepsilon} X_{k}\right)>\epsilon\right)<\infty .
$$

That is, (27) is established.

Proof of Theorem 2. We first prove that

$$
\sum_{n=1}^{\infty} \mathbb{V}\left(S_{n}>\epsilon n^{\alpha}\right)<\infty, \quad \forall \epsilon>0 .
$$

For any $\quad c>0$, $h_{c}(x):=-c I(x<-c)+x I(|x| \leq c)+c I(x>c)$, for all $n \geq 1,1 \leq k \leq n$, and let

$$
\begin{aligned}
Y_{n k}:= & h_{n^{\alpha}}\left(X_{k}\right)=-n^{\alpha} I\left(X_{k}<-n^{\alpha}\right) \\
& +X_{k} I\left(\left|X_{k}\right| \leq n^{\alpha}\right)+n^{\alpha} I\left(X_{k}>n^{\alpha}\right), \\
Z_{n k}:= & X_{k}-Y_{n k}=\left(X_{k}+n^{\alpha}\right) I\left(X_{k}<-n^{\alpha}\right) \\
& +\left(X_{k}-n^{\alpha}\right) I\left(X_{k}>n^{\alpha}\right) .
\end{aligned}
$$

Then, $\left\{Y_{n k} ; 1 \leq k \leq n, n \geq 1\right\}$ is also a sequence of upper extended negatively dependent random variables by $h_{c}(x) \in C_{l \text {,Lip }}$ and $h_{c}(x)$ being nondecreasing. Note that

$$
S_{n}=\sum_{k=1}^{n}\left(Y_{n k}-\widehat{\mathbb{E}} Y_{n k}\right)+\sum_{k=1}^{n} Z_{n k}+\sum_{k=1}^{n} \widehat{\mathbb{E}} Y_{n k}:=U_{n 1}+U_{n 2}+U_{n 3} .
$$

In order to prove (55), it suffices to verify that

$$
\sum_{n=1}^{\infty} \mathbb{V}\left(U_{n i}>\epsilon n^{\alpha}\right)<\infty \quad \text { for any } \epsilon>0, i=1,2 .
$$

$$
\frac{U_{n 3}}{n^{\alpha}} \longrightarrow 0 \quad \text { as } n \longrightarrow \infty
$$
have

We first prove that (59). For any $0<a<b$, by (37), we

$$
\begin{gathered}
I(a<x \leq b)=I(x \leq b)-I(x \leq a) \leq g\left(\frac{\mu x}{b}\right)-g\left(\frac{x}{a}\right), \quad(60) \\
I(-b \leq x<-a)=I\left(\frac{-x}{b} \leq 1\right)-I\left(\frac{-x}{a} \leq 1\right) \leq g\left(\frac{-\mu x}{b}\right)-g\left(\frac{-x}{a}\right) .
\end{gathered}
$$

For every $n$, there exists a $k$ such that $2^{k-1} \leq n<2^{k}$; thus, for any $r>0$, by $g(x) \downarrow, c_{r}$ inequality, (37) and (60), and (61), we obtain

$$
\begin{aligned}
\left|Y_{n 1}\right|^{r} \ll & \left|X_{1}\right|^{r} I\left(\left|X_{1}\right| \leq n^{\alpha}\right)+n^{\alpha r} I\left(\left|X_{1}\right|>n^{\alpha}\right) \\
\leq & +\left|X_{1}\right|^{r}\left(I\left(1<X_{1} \leq 2^{\alpha k}\right)+I\left(-2^{\alpha k} \leq X_{1}<1\right)\right) \\
& +2^{\alpha r k}\left(I\left(X_{1}>2^{\alpha(k-1)}\right)+I\left(-X_{1}>2^{\alpha(k-1)}\right)\right) \\
= & 1+\sum_{j=1}^{k}\left|X_{1}\right|^{r}\left(I\left(2^{\alpha(j-1)}<X_{1} \leq 2^{\alpha j}\right)+I\left(-2^{\alpha j} \leq X_{1}<-2^{\alpha(j-1)}\right)\right) \\
& +2^{\alpha r k}\left(I\left(X_{1}>2^{\alpha(k-1)}\right)+I\left(-X_{1}>2^{\alpha(k-1)}\right)\right) \\
& \leq 1+\sum_{j=1}^{k}\left|X_{1}\right|^{r}\left\{\left(g\left(\frac{\mu X_{1}}{2^{\alpha j}}\right)-g\left(\frac{X_{1}}{2^{\alpha(j-1)}}\right)\right)+\left(g\left(\frac{-\mu X_{1}}{2^{\alpha j}}\right)-g\left(\frac{-X_{1}}{2^{\alpha(j-1)}}\right)\right)\right\} \\
& +2^{\alpha r k}\left\{\left(1-g\left(\frac{X_{1}}{2^{\alpha(k-1)}}\right)\right)+\left(1-g\left(\frac{-X_{1}}{2^{\alpha(k-1)}}\right)\right)\right\} .
\end{aligned}
$$


Hence, by (5) and (37) and $g\left(\mu X_{1} / 2^{\alpha j}\right)-g\left(X_{1} /\right.$ $\left.2^{\alpha(j-1)}\right) \leq I\left(\mu 2^{\alpha(j-1)} \leq X_{1} \leq \mu^{-1} 2^{\alpha j}\right), g\left(-\mu X_{1} / 2^{\alpha j}\right)-g\left(-X_{1} /\right.$ $\left.2^{\alpha(j-1)}\right) \leq I\left(-\mu^{-1} 2^{\alpha j} \leq X_{1} \leq-\mu 2^{\alpha(j-1)}\right)$,

$$
\begin{aligned}
\widehat{\mathbb{E}}\left(\left|Y_{n 1}\right|^{r}\right) \ll & +\sum_{j=1}^{k}\left\{\widehat{\mathbb{E}}\left|X_{1}\right|^{r}\left(g\left(\frac{\mu X_{1}}{2^{\alpha j}}\right)-g\left(\frac{X_{1}}{2^{\alpha(j-1)}}\right)\right)+\widehat{\mathbb{E}}\left|X_{1}\right|^{r}\left(g\left(\frac{-\mu X_{1}}{2^{\alpha j}}\right)-g\left(\frac{-X_{1}}{2^{\alpha(j-1)}}\right)\right)\right\} \\
& +2^{\alpha r k}\left\{\widehat{\mathbb{E}}\left(1-g\left(\frac{X_{1}}{2^{\alpha(k-1)}}\right)\right)+\widehat{\mathbb{E}}\left(1-g\left(\frac{-X_{1}}{2^{\alpha(k-1)}}\right)\right)\right\} \\
\leq & 1+\sum_{j=1}^{k}\left(\mu^{-r} 2^{\alpha r j} \mathbb{V}\left(\left|X_{1}\right| \geq \mu 2^{\alpha(j-1)}\right)+\mu^{-r} 2^{\alpha r j} \mathbb{V}\left(\left|X_{1}\right| \geq \mu 2^{\alpha(j-1)}\right)\right) \\
& +2^{\alpha r k}\left(\mathbb{V}\left(X_{1}>\mu 2^{\alpha(k-1)}\right)+\mathbb{V}\left(X_{1}<-\mu 2^{\alpha(k-1)}\right)\right) \\
\ll & 1+\sum_{j=1}^{k} 2^{\alpha r j} \mathbb{V}\left(\left|X_{1}\right| \geq \mu 2^{-\alpha} 2^{\alpha j}\right)+2^{\alpha r k} \mathbb{V}\left(\left|X_{1}\right|>\mu 2^{-\alpha} 2^{\alpha k}\right) \\
\ll & \sum_{j=0}^{k} 2^{\alpha r j} \mathbb{V}\left(\left|X_{1}\right| \geq \mu 2^{-\alpha} 2^{\alpha j}\right) .
\end{aligned}
$$

Therefore, by $n^{-\alpha+1} \downarrow 0$ from $\alpha>1$, for $2^{k-1} \leq n<2^{k}$, we obtain

$$
\begin{aligned}
n^{-\alpha}\left|U_{n 3}\right| & \leq n^{1-\alpha} \widehat{\mathbb{E}}\left(\left|Y_{n 1}\right|\right) \leq 2^{(k-1)(1-\alpha)} \widehat{\mathbb{E}}\left(\left|Y_{n 1}\right|\right) \ll 2^{(1-\alpha) k} \sum_{j=0}^{k} 2^{\alpha j} \mathbb{V}\left(\left|X_{1}\right| \geq \mu 2^{-\alpha} 2^{\alpha j}\right) \\
& :=U_{n 31} .
\end{aligned}
$$

Next, we estimate $U_{n 31}$. Noting that, by (13),

$\sum_{j=0}^{\infty} \frac{2^{\alpha j}}{2^{(\alpha-1) j}} \mathbb{V}\left(\left|X_{1}\right|>2^{\alpha(j-1)}\right)=\sum_{j=0}^{\infty} 2^{j} \mathbb{V}\left(\left|X_{1}\right|>2^{-\alpha} 2^{\alpha j}\right)<\infty$.

It follows that

$$
U_{n 31} \longrightarrow 0, \quad \text { as } n \longrightarrow \infty,
$$

from $2^{(\alpha-1) j \uparrow \infty}$ and the Kronecker lemma. This combination, (59) and (64), was established.

Since $\left\{Y_{n k}-\widehat{\mathbb{E}} Y_{n k}, n \geq 1, k \leq n\right\}$ is also upper extended negatively dependent with $\widehat{\mathbb{E}}\left(Y_{n k}-\widehat{\mathbb{E}} Y_{n k}\right)=0$. It follows from Lemma 1, (63), and (13) that

$$
\begin{aligned}
\sum_{n=1}^{\infty} \mathbb{V}\left(U_{n 1}>\epsilon n^{\alpha}\right) & \ll \sum_{n=1}^{\infty} n^{-2 \alpha} \sum_{k=1}^{n} \widehat{\mathbb{E}} Y_{n k}^{2} \\
& =\sum_{n=1}^{\infty} n^{1-2 \alpha} \widehat{\mathbb{E}} Y_{n 1}^{2}=\sum_{k=1}^{\infty} \sum_{2^{k-1} \leq n<2^{k}} n^{1-2 \alpha} \widehat{\mathbb{E}} Y_{n 1}^{2}
\end{aligned}
$$

$$
\begin{aligned}
& \ll \sum_{k=1}^{\infty} \sum_{2^{k-1} \leq n<2^{k}} 2^{(k-1)(1-2 \alpha)} \sum_{j=0}^{k} 2^{2 \alpha j} \mathbb{V}\left(\left|X_{1}\right| \geq \mu 2^{-\alpha} 2^{\alpha j}\right) \\
& \ll \sum_{k=1}^{\infty} 2^{2(1-\alpha)} \sum_{j=0}^{k} 2^{2 \alpha j} \mathbb{V}\left(\left|X_{1}\right| \geq \mu 2^{-\alpha} 2^{\alpha j}\right) \\
& \ll \sum_{j=0}^{\infty} 2^{2 \alpha j} \sum_{k=j}^{\infty} 2^{2(1-\alpha) k} \mathbb{V}\left(\left|X_{1}\right|>\mu 2^{-\alpha} 2^{\alpha j}\right) \\
& \ll \sum_{j=0}^{\infty} 2^{2 j} \mathbb{V}\left(\left|X_{1}\right|>\mu 2^{-\alpha} 2^{\alpha j}\right) \\
& <\infty .
\end{aligned}
$$

By (5), (12), and (37), 


$$
\begin{aligned}
\sum_{n=1}^{\infty} \mathbb{V}\left(U_{n 2}>\epsilon n^{\alpha}\right) & \ll \sum_{n=1}^{\infty} \mathbb{V}\left(\sum_{k=1}^{n}\left|Z_{k}\right|>\epsilon n^{\alpha}\right) \\
& \leq \sum_{n=1}^{\infty} \mathbb{V}\left(\text { there exists a } k \in[1, n] \text { such that } X_{k}>n^{\alpha} \text { or } X_{k}<-n^{\alpha}\right) \\
& \leq \sum_{n=1}^{\infty} \sum_{k=1}^{n}\left(\mathbb{V}\left(X_{k}>n^{\alpha}\right)+\mathbb{V}\left(X_{k}<-n^{\alpha}\right)\right) \\
& \leq \sum_{n=1}^{\infty} \sum_{k=1}^{n}\left(\widehat{\mathbb{E}}\left[1-g\left(\frac{X_{k}}{n^{\alpha}}\right)\right]+\widehat{\mathbb{E}}\left[1-g\left(\frac{-X_{k}}{n^{\alpha}}\right)\right]\right) \\
& =\sum_{n=1}^{\infty} n\left(\widehat{\mathbb{E}}\left[1-g\left(\frac{X_{1}}{n^{\alpha}}\right)\right]+\widehat{\mathbb{E}}\left[1-g\left(\frac{-X_{1}}{n^{\alpha}}\right)\right]\right) \\
& \leq \sum_{n=1}^{\infty} n \mathbb{V}\left(\left|X_{1}\right|>\mu n^{\alpha}\right) \\
& <\infty .
\end{aligned}
$$

From this combination, (67), we get (58). Therefore, (55) holds.

Because of $\left\{-X_{n} ; n \geq 1\right\}$, also satisfying the conditions of Theorem 2 and considering $\left\{-X_{n} ; n \geq 1\right\}$ instead of $\left\{X_{n} ; n \geq 1\right\}$ in (55), we can obtain

$$
\sum_{n=1}^{\infty} \mathbb{V}\left(-S_{n}>\epsilon n^{\alpha}\right)=\sum_{n=1}^{\infty} \mathbb{V}\left(S_{n}<-\epsilon n^{\alpha}\right)<\infty
$$

Hence,

$$
\sum_{n=1}^{\infty} \mathbb{V}\left(\left|S_{n}\right|>\epsilon n^{\alpha}\right) \leq \sum_{n=1}^{\infty} \mathbb{V}\left(S_{n}>\epsilon n^{\alpha}\right)+\sum_{n=1}^{\infty} \mathbb{V}\left(S_{n}<-\epsilon n^{\alpha}\right)<\infty
$$

namely, (30) is established. This completes the Proof of Theorem 2.

\section{Data Availability}

No data were used to support this study.

\section{Conflicts of Interest}

The authors declare no conflicts of interest.

\section{Acknowledgments}

This research was supported by the National Natural Science Foundation of China (12061028) and Support Program of the Guangxi China Science Foundation (2018GXNSFAA281011).

\section{References}

[1] S. G. Peng, "BSDE and related g-expectation," Pitman Research Notes in Mathematics Series, vol. 364, pp. 141-159, 1997.
[2] S. G. Peng, "Monotonic limit theorem of BSDE and nonlinear decomposition theorem of Doob-Meyers type," Probability Theory and Related Fields, vol. 113, no. 4, pp. 473-499, 1999.

[3] S. G. Peng, "G-expectation, G-Brownian motion and related stochastic calculus of Ito type," 2006.

[4] S. G. Peng, "Multi-dimensional G-Brownian motion and related stochastic calculus under G-expectation," Stochastic Processes and Their Applications, vol. 118, no. 12, pp. 22232253, 2008.

[5] S. G. Peng, "Survey on normal distributions, central limit theorem, Brownian motion and the related stochastic calculus under sublinear expectations," Science in China Series A: Mathematics, vol. 52, no. 7, pp. 1391-1411, 2009.

[6] L. Denis and C. Martini, "A theoretical framework for the pricing of contingent claims in the presence of model uncertainty," National Academy of Sciences, vol. 16, no. 2, pp. 827-852, 2006.

[7] M. Marinacci, "Limit laws for non-additive probabilities and their frequentist interpretation," Journal of Economic Theory, vol. 84, no. 2, pp. 145-195, 1999.

[8] L. X. Zhang, "Strong limit theorems for extended independent and extended negatively dependent random variables under non-linear expectations," Science China Mathematics, vol. 23, 2016a.

[9] L. X. Zhang, "Exponential inequalities under the sub-linear expectations with applications to laws of the iterated logarithm," Science China Mathematics, vol. 59, no. 2, pp. 2503-2526, 2016b.

[10] L. X. Zhang, "Rosenthal's inequalities for independent and negatively dependent random variables under sub-linear expectations with applications," Science China Mathematics, vol. 59, no. 4, pp. 751-768, 2016c.

[11] C. Hu, "A strong law of large numbers for sub-linear expectation under a general moment condition," Statistics \& Probability Letters, vol. 119, pp. 248-258, 2016.

[12] Z. Chen, "Strong laws of large numbers for sub-linear expectations," Science China Mathematics, vol. 59, no. 5, pp. 945-954, 2016. 
[13] Q. Y. Wu and Y. Y. Jiang, "Strong law of large numbers and Chover's law of the iterated logarithm under sub-linear expectations," Journal of Mathematical Analysis and Applications, vol. 460, no. 1, pp. 252-270, 2018.

[14] Q. Y. Wu and J. F. Lu, "Another form of Chover's law of the iterated logarithm under sub-linear expectations. Revista de la Real Academia de Ciencias Exactas, Físicas y Naturales," Serie A. Matemáticas, January, vol. 114, no. 1, pp. 1-22, 2020.

[15] P. L. Hsu and H. Robbins, "Complete convergence and the law of large numbers," Proceedings of the National Academy of Sciences, vol. 33, no. 2, pp. 25-31, 1947.

[16] Q. Y. Wu, "Complete convergence for negatively dependent sequences of random variables," Journal of Inequalities and Applications, vol. 2010, Article ID 507293, 10 pages, 2010.

[17] T.-C. Hu, A. Rosalsky, and A. Volodin, "A complete convergence theorem for row sums from arrays of rowwise independent random elements in rademacher TypepBanach spaces," Stochastic Analysis and Applications, vol. 30, no. 2, pp. 343-353, 2012.

[18] X. J. Wang, X. Deng, L. L. Zheng, and S. H. Hu, "Complete convergence for arrays of rowwise negatively superadditivedependent random variables and its applications," Statistics, vol. 48 , no. 4 , pp. 834-850, 2014.

[19] X. J. Wang, A. T. Shen, Z. Y. Chen, and S. H. Hu, "Complete convergence for weighted sums of NSD random variables and its application in the EV regression model," TEST, vol. 24, no. 1, pp. 166-184, 2015.

[20] C. C. Liu, M. L. Guo, and D. Zhu, "Equivalent conditions of complete convergence for weighted sums of sequences of extended negatively dependent random variables," Communications in Mathematical Research, vol. 31, no. 1, pp. 40-50, 2015.

[21] P. Y. Chen and S. H. Sung, "Complete convergence and strong laws of large numbers for weighted sums of negatively orthant dependent random variables," Acta Mathematica Hungarica, vol. 148, no. 1, pp. 83-95, 2016.

[22] X. L. Tan, H. Wang, and Y. Zhang, "Complete convergence of the non-identically distributed pairwise NQD random sequences," Communications in Statistics-Theory and Methods, vol. 45, no. 9, pp. 2626-2637, 2016.

[23] Q. Y. Wu and Y. Y. Jiang, "Complete convergence and complete moment convergence for negatively associated sequences of random variables," Journal of Inequalities and Applications, vol. 2016, no. 1, 157 pages, 2016.

[24] A. T. Shen, M. X. Xue, and W. J. Wang, "Complete convergence for weighted sums of extended negatively dependent random variables," Communications in Statistics - Theory and Methods, vol. 46, no. 3, pp. 1433-1444, 2017.

[25] D. L. Yu and Q. Y. Wu, "Complete convergence for weighted sums of extended negatively dependent random variables under sub-linear expectations," Communications in Statistics-Theory and Methods, vol. 47, no. 19, pp. 4741-4750, 2018.

[26] W. J. Wang and Q. Y. Wu, "Complete convergence for arrays of row-wise ND random variables under sub-linear expectations," Communications in Statistics-Theory and Methods, vol. 48, no. 13, pp. 3165-3176, 2019.

[27] X. Deng and X. J. Wang, "On complete convergence for extended independent random variables under sub-linear expectations," National Academy of Sciences, vol. 57, no. 3, pp. $553-570,2020$. 